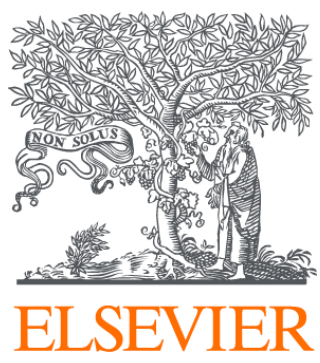

Since January 2020 Elsevier has created a COVID-19 resource centre with free information in English and Mandarin on the novel coronavirus COVID-

19. The COVID-19 resource centre is hosted on Elsevier Connect, the company's public news and information website.

Elsevier hereby grants permission to make all its COVID-19-related research that is available on the COVID-19 resource centre - including this research content - immediately available in PubMed Central and other publicly funded repositories, such as the WHO COVID database with rights for unrestricted research re-use and analyses in any form or by any means with acknowledgement of the original source. These permissions are granted for free by Elsevier for as long as the COVID-19 resource centre remains active. 


\title{
What challenges do UK adults face when adhering to COVID-19-related instructions? Cross-sectional survey in a representative sample
}

\author{
Chris Keyworth $^{\mathrm{a}, \mathrm{b}, *}$, Tracy Epton ${ }^{\mathrm{a}}$, Lucie Byrne-Davis ${ }^{\mathrm{c}}$, Jessica Z. Leather ${ }^{\mathrm{a}, \mathrm{b}}$, \\ Christopher J. Armitage ${ }^{a, b, d}$ \\ ${ }^{a}$ Manchester Centre for Health Psychology, School of Health Sciences, The University of Manchester, M13 9PL, United Kingdom \\ ${ }^{\mathrm{b}}$ NIHR Greater Manchester Patient Safety Translational Research Centre, The University of Manchester, Manchester Academic Health Science Centre, Manchester M13 \\ 9PL, United Kingdom \\ ${ }^{\mathrm{c}}$ Division of Medical Education, School of Medical Sciences, The University of Manchester, M13 9PL, United Kingdom \\ ${ }^{\mathrm{d}}$ Manchester University NHS Foundation Trust, Manchester Academic Health Science Centre, Manchester M13 9PL, United Kingdom
}

\section{A R T I C L E I N F O}

\section{Keywords:}

COVID-19

Prevention

Survey

Quantitative

\begin{abstract}
A B S T R A C T
Adherence to government COVID-19-related instructions is reported to be high, but the psychosocial impacts of measures such as self-isolation and physical distancing could undermine long-term adherence to containment measures. The first step in designing interventions to mitigate the impacts of adhering to COVID-19-related instructions is to identify what are the most prevalent challenges and what characterises the people facing them.

A cross-sectional survey was administered to a representative sample of the UK population $(N=2252)$, of whom $n=2139$ (94.9\%) reported adhering to the UK government's COVID-19-related instructions, and were included in the final analysis. Data were analysed using descriptive statistics and binary logistic regression.

Of the people who reported adhering to UK government's COVID-19-related instructions, 80.3\% reported experiencing challenges. Adults aged 55 years or over $(\mathrm{OR}=1.939,95 \% \mathrm{CI} 1.331-2.825)$ and $\mathrm{men}(\mathrm{OR}=0.489$, $95 \%$ CI $0.393-0.608$ ) were least likely to report challenges. Adjusting to changes in daily routine (reported by $48.7 \%$ of the sample), mental health (reported by $41.4 \%$ of the sample) and physical health (reported by $31.5 \%$ of the sample) were the most prevalent challenges.

For the first time, the present study quantifies the extent to which people experienced challenges when adhering to government COVID-19-related instructions. Few people reported experiencing no challenges when adhering to COVID-19-related instructions. Interventions to address the effects of changes in daily routine, mental health challenges, and physical health challenges should be prioritised, with a focus on key subgroups including women, younger adults, and people without care commitments.
\end{abstract}

\section{Introduction}

Until widescale rollout of effective vaccines for COVID-19, performing behaviours (e.g., hand washing, self-isolating when appropriate, physical distancing) to mitigate transmission is the only way to control the spread of the virus. Public adherence to UK government COVID-19-related instructions is high (Armitage et al., in press), yet the wide-ranging impacts on health and wellbeing of the COVID-19 pandemic and its associated containment measures are well documented. These include heightened levels of stress, depression and anxiety, low mood and sleep-related disturbances (Gao et al., 2020; Banks and Xu, 2020; Brooks et al., 2020; Gualano et al., 2020; Lauri Korajlija and Jokic-Begic, 2020). People may also face financial challenges due to being unable to work (Brooks et al., 2020), changes in daily routines and structure (Williams et al., 2020), and lack of access to childcare (Dalton et al., 2008). Failing to address these challenges may undermine the sustained changes in behaviour that are needed to tackle the pandemic. However, previous research is limited by its focus on qualitative studies using small unrepresentative samples only, or being rapid reviews, as opposed to systematic reviews. To address this limitation, there is a need to understand the challenges faced specifically when adhering to government COVID-19-related instructions using a nationally

\footnotetext{
* Corresponding author at: Manchester Centre for Health Psychology, School of Health Sciences, University of Manchester, M13 9PL, United Kingdom.

E-mail addresses: Chris.keyworth@manchester.ac.uk (C. Keyworth), tracy.epton@manchester.ac.uk (T. Epton), lucie.byrne-davis@manchester.ac.uk (L. ByrneDavis), jessica.leather@manchester.ac.uk (J.Z. Leather), chris.armitage@manchester.ac.uk (C.J. Armitage).
} 
representative sample.

A first step in developing interventions to mitigate these psychosocial challenges is to understand the extent of the various challenges and at whom such interventions need to be targeted. The present study aimed to: (1) assess the challenges experienced as a result of adhering to the UK government's COVID-19 related instructions (limiting time spent outside the home, staying $2 \mathrm{~m}$ away from people outside the household at all times, and maintaining hand hygiene), and (2) identify the people who may need targeted help to deal with such challenges with a view to bolstering adherence to the UK government's COVID-19-related instructions. With respect to the latter, recent findings show the disproportionate effects of COVID-19 on people with lower incomes (Blundell et al., 2020), people from Black, Asian and minority ethnic (BAME) groups (PHE, 2020), and people of lower socioeconomic status (PratsUribe et al., 2020). Other sociodemographic variables, including age, gender, and education level may also be associated with specific challenges experienced during pandemics (Taylor et al., 2008; Qiu et al., 2020). For example, women, younger adults and those with lower levels of educational qualifications are more vulnerable to high psychological distress (Taylor et al., 2008; Horesh et al., 2020). It is important to understand more about sociodemographic variables as these may affect levels of compliance within the population. For example, psychological traits such as self-efficacy, psychosocial variables such as perceived capability opportunity and motivation to adhere to government guidelines, and demographic factors such as younger age and higher education level are shown to be associated with decreased compliance of protective health measures (Armitage et al., in press; Roma et al., 2020a; Nivette et al., 2021). We therefore aimed to explore these further. Based on previous evidence it is predicted that COVID-19-related challenges will be more prevalent in women, older adults, people from BAME groups, and people of lower socioeconomic status.

\section{Methods}

\subsection{Design and procedure}

A cross-sectional survey design was used. A sample of 2252 UK adults were recruited via a survey panel company (YouGov) on 30th April 2020, when the UK had been in lockdown for 5 weeks. A representative sample of the UK population were invited to take part in an online questionnaire, administered as part of a daily "omnibus" survey. Participants were incentivised in accordance with YouGov's points system, whereby respondents accumulate points for taking part in online surveys. The data were collated by YouGov and sent securely to the research team for analysis. Ethical approval was obtained from a University Research Ethics Committee (ref: 2020-9551-15,105) and participants gave informed consent at the beginning of the survey.

\section{Measures}

\subsection{Sociodemographic variables}

The questionnaire, as part of a wider survey (Armitage et al., in press), included sociodemographic variables of age, gender, ethnicity and social grade (type of occupation: manual, non-manual/ unemployed) according to UK Office for National Statistics measures (Statistics UOfN, 2020) ${ }^{1}$.

\subsection{Capturing experiences of adherence}

Adherence to government COVID-19-related instructions was measured using a single item, "How closely are you following the UK

\footnotetext{
${ }^{1}$ Other items analysed from the same Covid-19 dataset are reported elsewhere see https://osf.io/ew82z/
}

government's COVID-19-related instructions?" that participants rated on an 11-point scale (not at all[0]-very much so[10]). The present study aimed to understand the challenges associated with adherence to government instructions; therefore, people who reported adherence were included in the analysis. ${ }^{2}$ Adherence was defined as participants scoring above the scale midpoint (scores $>5$ ); all participants scoring $\leq 5$ were excluded from the analysis. This method was deemed appropriate in order to eliminate neutral responses (i.e. on the scale midpoint), and to ensure that we identified people with at least modest exposure to the challenges of adhering to the UK government's COVID-19-related instructions. Of the total sample $(N=2252), 2139$ (94.9\%) people reported adherence $>5(0-10$ scale $)$ and were included in the final analysis.

\subsection{Challenges faced when following COVID-19-related instructions}

Participants were also asked a single item "which, if any, of the following challenges are you facing in following the UK government's coronavirus (COVID-19) related instructions?" They were presented with a list of seven response options with respect to challenges identified in the literature, alongside descriptions of the natures of the challenges. Response options were: employment challenges (e.g. being unable to work, being made redundant, furlough etc.) (Thomas et al., 2015), care commitment challenges (e.g. childcare or looking after elderly relatives etc.) (Andrew et al., 2020), physical health challenges (e.g. lack of exercise, unusual aches and pains etc.) (Williams et al., 2020), adjusting to changes to your usual daily routine (Williams et al., 2020), financial challenges (e.g. paying bills, rent, accessing the benefit system etc.) (Brooks et al., 2020), mental health challenges (e.g. anxiety, feeling down, loneliness etc.) (Gao et al., 2020), environmental challenges (e.g. lack of space at home, no access to outside space etc.) (Thomas et al., 2015). Using individual checkboxes, participants were asked to identify which of the challenges they were currently facing (participants were free to choose as many as they felt were applicable to them personally). For those not facing any challenges a further option was provided: "not applicable - I'm not facing any challenges with government instructions for coronavirus".

\subsection{Analyses}

Data were weighted to be reflective of the UK population. Whilst the residuals were non normally distributed, the methods (including the large sample size) were considered robust; logistic regression can be used irrespective of distribution (Schreiber-Gregory et al., 2018). Descriptive statistics were used to quantify the challenges experienced adhering to government COVID-19-related instructions. Binary logistic regression was used to examine correlates of COVID-19-related challenges, according to sociodemographic variables (gender, age, ethnicity, social grade, and whether respondents were either a parent or a guardian; all dummy coded). Each challenge was recorded as a binary outcome (challenge experienced[1] or challenge not experienced $[0]$ ).

\section{Results}

\subsection{Participant characteristics}

Participant characteristics are presented in Table 1. The mean age of participants was 48.46 years $(S D=17.52)$. Most participants were of

\footnotetext{
${ }^{2}$ Preliminary analysis showed challenges overall were more prevalent in people who reported adherence $(n=2139)$, compared to those who reported non-adherence $(n=113)$ : mental health $(41.4 \%$ vs. $28.3 \%)$, physical health ( $31.5 \%$ vs $20.4 \%$ ), daily routine ( $48.7 \%$ vs. $32.7 \%$ ), care commitments ( 12.9 vs. 5.3), environmental ( $19.5 \%$ vs. $18.6 \%)$, financial ( $14.0 \%$ vs. $10.6 \%)$, employment $(19.5 \%$ vs. $18.6 \%)$.
} 
Table 1

Sample characteristics, adherence and challenges faced when adhering to government COVID-19-related instructions.

\begin{tabular}{|c|c|c|c|c|}
\hline Variable & $N$ & $(\%)$ & Mean & $S D$ \\
\hline \multicolumn{5}{|l|}{ Gender } \\
\hline Men & 1010 & 47.2 & - & - \\
\hline Women & 1129 & 52.8 & - & - \\
\hline Age & - & - & 48.46 & 17.52 \\
\hline \multicolumn{5}{|l|}{ Social grade } \\
\hline Non-manual worker & 1225 & 57.2 & - & - \\
\hline Manual / unemployed & 915 & 42.8 & - & - \\
\hline \multicolumn{5}{|l|}{ Ethnicity } \\
\hline White & 2000 & 93.5 & - & - \\
\hline Black, Asian, minority ethnic/prefer not to say & 139 & 6.5 & - & - \\
\hline \multicolumn{5}{|l|}{ Adherence } \\
\hline $\begin{array}{l}\text { "How closely are you following the UK } \\
\text { government's COVID-19-related } \\
\text { instructions?" (not at all[0]-very much so[10]). }\end{array}$ & - & - & 9.09 & 1.10 \\
\hline Challenges & & & $1.81^{\mathrm{a}}$ & $1.54^{\mathrm{a}}$ \\
\hline Adjusting to changes to daily routine & 1043 & 48.7 & - & - \\
\hline Mental health challenges & 886 & 41.4 & - & - \\
\hline Physical health challenges & 673 & 31.5 & - & - \\
\hline Employment challenges & 417 & 19.5 & - & - \\
\hline Financial challenges & 299 & 14.0 & - & - \\
\hline Environmental challenges & 288 & 13.5 & - & - \\
\hline Care commitment challenges & 276 & 12.9 & - & - \\
\hline Other & 145 & 6.8 & - & - \\
\hline $\begin{array}{l}\text { Not applicable "I'm not facing any challenges } \\
\text { with government instructions for } \\
\text { coronavirus" }\end{array}$ & 422 & 19.7 & - & - \\
\hline Don't know / prefer not to say & 66 & 3.1 & - & - \\
\hline
\end{tabular}

${ }^{a}$ Refers to the average number of challenges identified (e.g. mental health challenges, physical health challenges).

white ethnic background ( $n=2000 ; 93.5 \%$ ) and $52.8 \%$ of the sample were women. Self-reported adherence to government-related instructions was high $(M=9.09, S D=1.10$ on a $0-10$ scale $)$.

\subsection{Challenges faced when following COVID-19-related instructions}

The most prevalent challenges faced when adhering to government COVID-19-related instructions (presented in Table 1) were: adjusting to changes in daily routine (reported by $48.7 \%$ of the sample), mental health challenges (reported by $41.4 \%$ of the sample), and physical health challenges (reported by $31.5 \%$ of the sample); $19.7 \%$ of the sample reported not facing any challenges with government instructions for COVID-19. Of the total sample, $430(20.1 \%)$ people reported experiencing both mental and physical health challenges. For those reporting one challenge only ( $n=496 ; 23.2 \%$ of the sample), $44.5 \%$ of these people reported challenges in adjusting to daily routines only, $21.4 \%$ reported mental health challenges only, and $12.8 \%$ reported physical challenges only. The mean number of specific challenges experienced was 1.81 , and $30.5 \%$ of the sample reported three or more challenges.

\subsection{Associations between sociodemographic variables and challenges faced}

Table 2 shows the binary logistic regression results of the prevalence of challenges when adhering to government-related-COVID-19 instructions, according to sociodemographic variables. Women compared with men (OR $=0.489,95 \% \mathrm{CI} 0.393-0.608$ ), and people younger than 55 were more likely to report challenges $(\mathrm{OR}=1.939$, 95\% CI 1.331-2.825). We found no evidence of differences in the likelihood of reporting challenges based on social grade and ethnicity.

Multiple challenges (i.e. more than one challenge) were reported more among women (OR $=1.604,95 \%$ CI 1.352-1.903) compared with men, and among people aged 25-34 years compared with people aged 18-24 years $(\mathrm{OR}=1.426,95 \% \mathrm{CI} 1.007-2.020)$. Multiple challenges were less prevalent among older adults aged 55 years or older compared with younger adults aged $18-24$ years $(\mathrm{OR}=0.520$, 95\%CI $0.388-0.696$ ), and among people who were parents or guardians ( $\mathrm{OR}=$ $0.824,95 \% \mathrm{CI}=0.694-0.978$ ).

Mental health challenges were reported more among women $(\mathrm{OR}=$ $1.790,95 \%$ CI 1.503-2.132) compared to men, among younger adults aged 18-24 years compared with those aged $45-54$ years $(\mathrm{OR}=0.579$, $95 \%$ CI $0.415-0.804)$ and 55 years or over (OR $=0.359$, 95\%CI $0.267-0.482$ ), and among people who were not parents or guardians $(\mathrm{OR}=0.610,95 \% \mathrm{CI} 0.512-0.726)$. Physical health challenges were more prevalent among women (OR $=1.354$, 95\%CI 1.126-1.628) compared to men, and among people who were not parents or guardians (OR $=0.753$, 95\%CI 0.629-0.902).

Challenges in adjusting to changes to daily routine were more prevalent in women $(\mathrm{OR}=1.665$, 95\%CI 1.403-1.976) compared with men, and more prevalent among people of higher social grade (OR = 1.186, 95\% CI 1.004-1.402) compared to lower social grade. Care commitment challenges were more prevalent in women $(\mathrm{OR}=1.627$, 95\%CI 1.253-2.112) compared with men, and in people aged 25-34 years (OR $=5.217$, 95\%CI 2.412-11.288), 35-44 years $(\mathrm{OR}=8.291$, 95\%CI 3.927-17.502), 45-54 years (OR 6.224, 95\%CI 2.920-13.263) and aged 55 years or over ( $\mathrm{OR}=2.601,95 \% \mathrm{CI} 1.231-5.494)$ than people aged 18-24 years, and more prevalent in people who were parents or guardians (OR $=2.618,95 \%$ CI 1.959-3.499).

Environmental challenges were more prevalent among people aged $18-24$ years, compared to those aged $35-44$ years $(\mathrm{OR}=0.592,95 \% \mathrm{CI}$ $0.377-0.838$ ), $45-54$ years (OR $=0.307,95 \%$ CI $0.195-0.484$ ), and 55 years or over ( $\mathrm{OR}=0.189,95 \% \mathrm{CI} 0.126-0.283$ ), and more prevalent among people who were not parents or guardians ( $\mathrm{OR}=0.457,95 \% \mathrm{CI}$ 0.355-0.588). Environmental challenges were more prevalent among people of higher social grade $(\mathrm{OR}=1.314,95 \% \mathrm{CI} 1.017-1.697$ ) compared to people of lower social grade.

Financial challenges were less prevalent among people aged 55 or over (OR $=0.306,95 \%$ CI $0.197-0.477)$ compared to people aged 18-24 years, and less prevalent among people of white ethnic background (OR $=0.519,95 \%$ CI $0.334-0.806$ ) compared with people of non-white ethnic background. Employment challenges were more prevalent among people who were aged 18-24 years, compared with people aged $35-44$ years (OR $=0.644,95 \%$ CI $0.448-0.924)$, 45-54 years $(\mathrm{OR}=0.679,95 \% \mathrm{CI}=0.472-0.976)$, and 55 years or over $(\mathrm{OR}=$ 0.224 , 95\%CI $0.157-0.320$ ), and more prevalent among people who were not parents or guardians ( $\mathrm{OR}=0.626,95 \% \mathrm{CI} 0.505-0.776$ ).

\section{Discussion}

The most prevalent challenges faced when adhering to government COVID-19-related instructions were: adjusting to changes in daily routines, mental health challenges, and physical health challenges. Demographic factors such as gender and age influenced the likelihood of experiencing COVID-19 related challenges during periods of lockdown. Contrary to our predictions, we found no evidence of greater challenges among people of lower social grade, and we found lower prevalence of challenges experienced by people from BAME groups.

\subsection{Comparison with previous studies}

Consistent with recent studies, our findings suggest that government COVID-19-related instructions such as social distancing and selfisolation pose a significant challenge to peoples' mental health and wellbeing (Gao et al., 2020; Banks and Xu, 2020; Brooks et al., 2020; Gualano et al., 2020; Lauri Korajlija and Jokic-Begic, 2020; Williams et al., 2020). We found evidence of high prevalence of self-reported mental and physical health challenges. We found that $40.8 \%$ of our sample reported experiencing mental health challenges, which far exceeds the one in six people presented in national statistics, who report experiencing a common mental health problem in a given week in England (McManus et al., 2016). Further, 20.1\% of people reported 


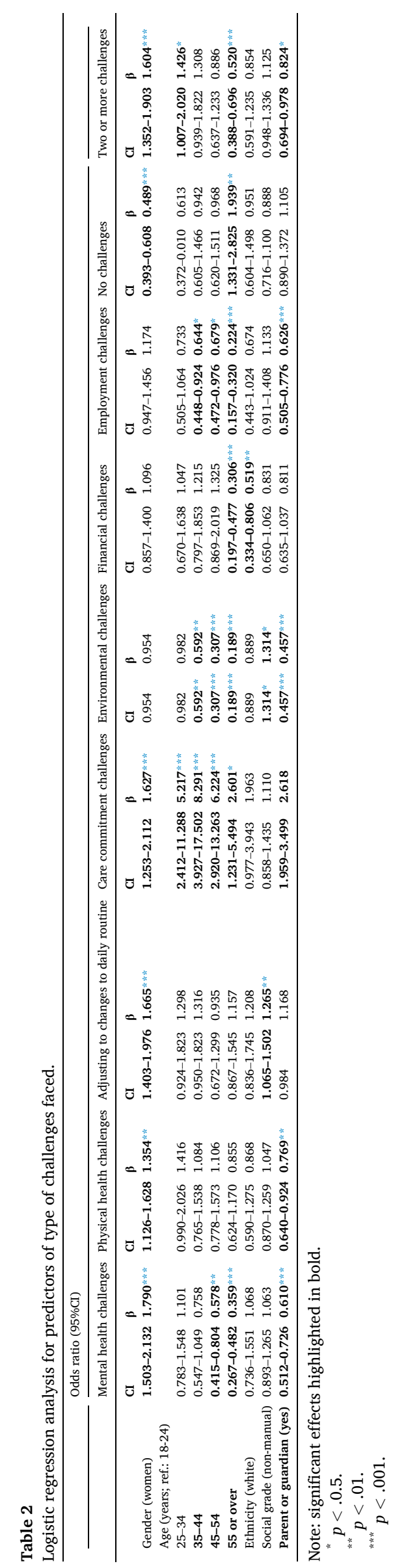

experiencing both mental and physical health challenges. The presence of both mental and physical health challenges is well documented; $30 \%$ of the UK population living with a long-term condition will also have a mental health problem (approximately 4.6 million people) (Naylor et al., 2012).

We found evidence that demographic factors influence the likelihood of the presence of COVID-19-related challenges, suggesting certain groups are more likely than others to experience specific challenges. Our findings suggest that groups known to be disproportionately affected by COVID-19, such as males, older adults, and people from BAME groups (PHE, 2020) do not appear to report higher prevalence of perceived challenges. Women were more likely than men to experience multiple challenges, and specifically with respect to physical and mental health challenges, and challenges related to care commitments and adjustments to daily routines. One possible explanation for this may be due to women being more likely than men to be primary caregivers or key workers, which may consequently result in significant psychological challenges (Horesh et al., 2020; Society, 2020). Further, there is evidence from recent studies that mothers, compared to fathers, are more likely to have lost or quit their job, or to have been furloughed since the start of lockdown, and carry the additional burden of spending less time on paid work, and more time on household responsibilities and childcare (Andrew et al., 2020; Hupkau and Petrongolo, 2020). Consistent with recent findings (Daly et al., 2020), mental health challenges were more prevalent in younger adults compared with older adults. Given the disproportionate effects of COVID-19 on people from Black, Asian and minority ethnic (BAME) groups (PHE, 2020), we expected higher prevalence of challenges experienced. However, people of non-white ethnic background were more likely than people of white ethnic background to experience financial challenges. This supports recent findings showing that compared to people of white British background, people of non-white ethnic background, including BAME and migrant groups, are more likely to experience COVID-19-related financial challenges including household income loss, or falling behind with bill payments (Hu, 2020). Given the relatively small number of people from BAME groups in our study ( $<6 \%$ of the total sample), it would therefore be valuable for future studies to explore the prevalence of specific challenges within sub-populations further using large samples. Another unexpected finding was that people of higher social grade were more likely than people of lower social grade to experience challenges in adjusting to daily routines and environmental challenges. These could be explained by specific changes to living circumstances, including a change in work environment (moving from office-based work to working at home), or having to work from home whilst looking after children. Further, key workers are more likely to be of lower social grade than higher social grade (Fernández-Reino et al., 2020), and may have not experienced the same shift in routine or environment (Blundell et al., 2020).

Conversely, certain demographic factors were protective factors for experiencing COVID-19-related challenges. People aged 35 years and older were less likely to experience environmental challenges and employment challenges, compared with younger adults who are more likely to: (a) live in non-rural areas than rural areas, with limited access to green space (Thomas et al., 2015), and (b) face disruption in education, and who were already facing great uncertainty in job markets prior to COVID-19 (Organization IL, 2020). People who were a parent or guardian were less likely to experience multiple challenges, and were less likely to experience mental and physical health challenges. This is consistent with research showing that having children is associated with reduced stress and anxiety, increased physical and psychological quality of life during COVID-19 (Horesh et al., 2020), and lower prevalence of depression (Roma et al., 2020b). One possible explanation may be that particularly people who are single or who have no children may experience loneliness, which is associated with adverse physical and psychological outcomes (Jaremka et al., 2013; Mazza et al., 2020) including feeling detached from sources of social support (Akin, 2010). These 
thoughts and feelings may be heightened during extended periods of social isolation. Conversely, having children may be a source of positive emotions and may serve to motivate parents to take preventive action, with childcare acting to divert attention away from negative emotions (Roma et al., 2020b). People who were a parent or guardian were also less likely to face environmental and employment challenges, which may be attributable to parents being able to carry on working at work and having the necessary space at home to educate their children (Blundell et al., 2020).

\subsection{Implications and future research}

Whilst people may report high levels of adherence to governmentrelated instructions during the pandemic (Armitage et al., in press), public health crises pose a significant challenge to many aspects of people's lives. Sociodemographic factors may influence the likelihood of compliance with protective health measures (Roma et al., 2020a; Nivette et al., 2021). By not addressing the challenges faced by particular groups within society, this may undermine the sustained changes in behaviour that are needed over the long-term. Due to a lack of tailored interventions promoting sustained adherence to COVID-19 containment measures, our findings suggest two important areas for public health policy makers. First, to encourage sustained adherence, public health communication messages should be tailored to specific groups, who may face different challenges and accessibility needs when adhering to protective health measures (British Psychological Society, 2020; Ghio et al., 2020). Second, specific characteristics of the population should be considered when designing interventions to address the challenges faced during pandemics identified in this study. Women, younger adults and people without care commitments in particular, would benefit from such interventions. Interventions would also benefit from considering people who report multiple challenges. Our findings suggest an urgent need to prioritise interventions which address the physical, psychological and social impacts of the pandemic. Future research must identify whether the challenges identified in our study persist over the long-term, such as mental and physical health outcomes, and the challenges of adjusting to new daily routines, including isolation, working arrangements including enforced remote working, caring responsibilities and home schooling (O'Connor et al., 2020; Hoffart et al., 2020; Holmes et al., 2020; Lee, 2020). Evidence from behavioural science should guide intervention development, such as using the behaviour change wheel to identify the specific content and function of interventions, and evaluate intervention options (Michie et al., 2020; Michie et al., 2011). Our findings highlight the need for specific interventions to address the challenges identified by specific groups in our study. These may include habit change interventions to support people in establishing new daily routines (Lally and Gardner, 2013), and greater investment in services to improve physical and mental health, such as the Improving Access to Psychological Therapy (IAPT) programme that can be delivered remotely (Rushton et al., 2019), remote consultations delivered by healthcare professionals to address physical health concerns (Greenhalgh et al., 2020), and home-based interventions to promote physical health (Ricci et al., 2020).

\section{Strengths and limitations}

One strength of the present study is that it was carried out among a large representative sample of the UK population. However, the crosssectional design means we were unable to monitor any changes in reported challenges over time. This is particularly important given the nature of the challenges experienced during different stages of a pandemic are likely to change as government measures are relaxed, and later reintroduced. Whilst the nature of the topic (i.e. asking people to report whether they were following government-related instructions) may, for some participants, result in socially desirable responses, we found that men were more likely than women to report no challenges.
This may suggest that men are underreporting perceived challenges or could reflect socio structural inequalities; further in-depth qualitative work would provide insight into this issue. Further, the use of a single item to measure adherence to government-related instructions may not fully capture the complexities of adherence, in light of rapidly changing government policies. Future studies would benefit from examining adherence to specific policies, as they are implemented.

\section{Conclusions}

The present study suggests an urgent need to consider the challenges people face when adhering to government instructions, particularly during periods of lockdown, when designing interventions to support the health and wellbeing of the population. This may include the design of new interventions, but also investment in existing services, particularly for mental health. Our findings suggest there are important subgroups that should be the focus of tailored interventions to promote sustained adherence to COVID-19 containment measures. Interventions should be targeted at women, younger adults and people without care commitments in particular. Addressing the challenges that people face are likely to promote sustained adherence to government instructions at the same time as promoting the health and wellbeing of the population during a health emergency.

\section{Credit author statement}

CK, CJA and TE conceived the study. CK was responsible data curation and formal analysis. CK and CJA wrote the original draft, and all authors reviewed, edited and commented on later drafts.

\section{Ethical approval and informed consent}

Ethical approval was obtained from a University Research Ethics Committee (ref: 2020-9551-15105) and participants gave informed consent at the beginning of the survey. the study was performed in accordance with the ethical standards as laid down in the 1964 Declaration of Helsinki and its later amendments or comparable ethical standards.

\section{Funding information}

This study was jointly funded by the NIHR Greater Manchester Patient Safety Translational Research Centre and the Manchester Centre for Health Psychology, and supported by the NIHR Manchester Biomedical Research Centre. NIHR had no role in the design of this study and did not have any role during its execution, analyses, interpretation, and storage of the data or decision to submit results.

\section{Declaration of competing interest}

The authors declare that they have no conflict of interest.

\section{References}

Akin, A., 2010. Self-compassion and loneliness. Int. Online J. Educat. Sci. 2 (3). Andrew, A., Cattan, S., Dias, M.C., Farquharson, C., Kraftman, L., Krutikova, S., et al., 2020. How are Mothers and Fathers Balancing Work and Family Under Lockdown? Institute for Fiscal Studies.

Armitage C., Keyworth C., Leather J., Byrne-Davis L., Epton T. (In Press) Identifying targets for interventions to support public adherence to government COVID-19related instructions. BMC Health Services Research.

Banks, J., Xu, X., 2020. The Mental Health Effects of the First Two Months of Lockdown and Social Distancing during the Covid-19 Pandemic in the UK. IFS Working Papers.

Blundell, R., Costa Dias, M., Joyce, R., Xu, X., 2020. COVID-19 and inequalities*. Fisc. Stud. 41 (2), 291-319.

Brooks, S.K., Webster, R.K., Smith, L.E., Woodland, L., Wessely, S., Greenberg, N., et al., 2020. The psychological impact of quarantine and how to reduce it: rapid review of the evidence. Lancet 395 (10227), 912-920. 
Dalton, C.B., Durrheim, D.N., Conroy, M.A., 2008. Likely impact of school and childcare closures on public health workforce during an influenza pandemic: a survey. Comm. Dis. Intelligence Quarterly Report. 32 (2), 261-262.

Daly, M., Sutin, A., Robinson, E., 2020. Longitudinal Changes in Mental Health and the COVID-19 Pandemic: Evidence from the UK Household Longitudinal Study.

Fernández-Reino, M., Sumption, M., Vargas-Silva, C., 2020. From low-skilled to key workers: the implications of emergencies for immigration policy. Oxf. Rev. Econ. Policy 36 (Supplement_1). S382-S96.

Gao, J., Zheng, P., Jia, Y., Chen, H., Mao, Y., Chen, S., et al., 2020. Mental health problems and social media exposure during COVID-19 outbreak. PLoS One 15 (4) e0231924-e.

Ghio, D., Lawes-Wickwar, S., Tang, M.Y., Epton, T., Howlett, N., Jenkinson, E., et al., 2020. What Influences people's Responses to Public Health Messages for Managing Risks and Preventing Disease during Public Health Crises? A Rapid Review of the Evidence and Recommendations.

Greenhalgh, T., Koh, G.C.H., Car, J., 2020. Covid-19: a remote assessment in primary care. BMJ. 368, m1182.

Gualano, M.R., Lo Moro, G., Voglino, G., Bert, F., Siliquini, R., 2020. Effects of Covid-19 lockdown on mental health and sleep disturbances in Italy. Int. J. Environ. Res. Public Health 17 (13), 4779.

Hoffart, A., Johnson, S.U., Ebrahimi, O.V., 2020. Loneliness and social distancing during the COVID-19 pandemic: Risk factors and associations with psychopathology. Frontiers in Psychiatry 11, 1297.

Holmes, E.A., O'Connor, R.C., Perry, V.H., Tracey, I., Wessely, S., Arseneault, L., et al., 2020. Multidisciplinary research priorities for the COVID-19 pandemic: a call for action for mental health science. Lancet Psychiatry 7 (6), 547-560.

Horesh, D., Kapel Lev-Ari, R., Hasson-Ohayon, I., 2020. Risk factors for psychological distress during the COVID-19 pandemic in Israel: Loneliness, age, gender, and health status play an important role. Br. J. Health Psychol. 25 (4), 925-933. https://doi. org/10.1111/bjhp.12455 n/a(n/a).

Hu, Y., 2020. Intersecting ethnic and native-migrant inequalities in the economic impact of the COVID-19 pandemic in the UK. Res. Soc. Stratificat. Mobil. 68, 100528.

Hupkau, C., Petrongolo, B., 2020. COVID-19 and gender gaps: latest evidence and lessons from the UK. VoxEU org 22.

Jaremka, L.M., Fagundes, C.P., Glaser, R., Bennett, J.M., Malarkey, W.B., KiecoltGlaser, J.K., 2013. Loneliness predicts pain, depression, and fatigue: understanding the role of immune dysregulation. Psychoneuroendocrinology. 38 (8), 1310-1317.

Lally, P., Gardner, B., 2013. Promoting habit formation. Health Psychol. Rev. 7 (Suppl. 1). S137-S58.

Lauri Korajlija, A., Jokic-Begic, N., 2020. COVID-19: concerns and behaviours in Croatia. Br. J. Health Psychol. 25 (4), 849-855. https://doi.org/10.1111/bjhp.12425.

Lee, J., 2020. Mental health effects of school closures during COVID-19. Lancet Child Adolesc Health. 4 (6), 421.

Mazza, C., Ricci, E., Biondi, S., Colasanti, M., Ferracuti, S., Napoli, C., et al., 2020. A Nationwide Survey of Psychological Distress among Italian People during the COVID-19 Pandemic: Immediate Psychological Responses and Associated Factors. Int. J. Environ. Res. Public Health 17 (9).

McManus, S., Bebbington, P., Jenkins, R., Brugha, T., 2016. Mental Health and Wellbeing in England: The Adult Psychiatric Morbidity Survey 2014: NHS Digital.

Michie, S., van Stralen, M.M., West, R., 2011. The behaviour change wheel: a new method for characterising and designing behaviour change interventions. Implementation Science: IS 6, 42.

Michie, S., West, R., Rogers, M.B., Bonell, C., Rubin, G.J., Amlôt, R., 2020. Reducing SARS-CoV-2 transmission in the UK: A behavioural science approach to identifying options for increasing adherence to social distancing and shielding vulnerable people. Br. J. Health Psychol. 25, 945-956. https://doi.org/10.1111/bjhp.12428.

Naylor, C., Parsonage, M., McDaid, D., Knapp, M., Fossey, M., Galea, A., 2012. LongTerm Conditions and Mental Health: The Cost of Co-Morbidities.
Nivette, A., Ribeaud, D., Murray, A., Steinhoff, A., Bechtiger, L., Hepp, U., et al., 2021. Non-compliance with COVID-19-related public health measures among young adults in Switzerland: insights from a longitudinal cohort study. Soc. Sci. Med. 268, 113370.

Organization IL, 2020. World Employment and social outlook - trends (2020). In: Contract No.: 10 September 2020.

PHE. Disparities in the risk and outcomes of Covid-19. Available from. https://assets. publishing.service.gov.uk/government/uploads/system/uploads/attachment_ data/file/908434/Disparities_in_the_risk_and_outcomes_of_COVID_August_2020_ update.pdf.

Prats-Uribe, A., Paredes, R., Prieto-Alhambra, D., 2020. Ethnicity, comorbidity, socioeconomic status, and their associations with COVID-19 infection in England: a cohort analysis of UK Biobank data. medRxiv, 2020.05.06.20092676.

Qiu, J., Shen, B., Zhao, M., Wang, Z., Xie, B., Xu, Y., 2020. A nationwide survey of psychological distress among Chinese people in the COVID-19 epidemic: implications and policy recommendations. General Psychiatry. 33 (2), e100213.

Ricci, F., Izzicupo, P., Moscucci, F., Sciomer, S., Maffei, S., Di Baldassarre, A., et al., 2020. Recommendations for physical inactivity and sedentary behavior during the coronavirus disease (COVID-19) pandemic. Front. Public Health 8, 199.

Roma, P., Monaro, M., Muzi, L., Colasanti, M., Ricci, E., Biondi, S., et al., 2020a. How to improve compliance with protective health measures during the COVID-19 outbreak: testing a moderated mediation model and machine learning algorithms. Int. J. Environ. Res. Public Health 17 (19), 7252.

Roma, P., Monaro, M., Colasanti, M., Ricci, E., Biondi, S., Di Domenico, A., et al., 2020b. A 2-month follow-up study of psychological distress among italian people during the COVID-19 lockdown. Int. J. Environ. Res. Public Health 17 (21).

Rushton, K., Fraser, C., Gellatly, J., Brooks, H., Bower, P., Armitage, C.J., et al., 2019. A case of misalignment: the perspectives of local and national decision-makers on the implementation of psychological treatment by telephone in the Improving Access to Psychological Therapies Service. BMC Health Serv. Res. 19 (1), 997.

Schreiber-Gregory, D., Jackson, H.M., Bader, K.S., 2018. Logistic and Linear Regression Assumptions: Violation Recognition and Control.

Society, F., 2020. Coronavirus: Urgent Call for UK Government to Support Women and Girls.

O'Connor, D.B., Aggleton, J.P., Chakrabarti, B., Cooper, C.L., Creswell, C., Dunsmuir, S., Fiske, S.T., Gathercole, S., Gough, B., Ireland, J.L., Jones, M.V., Jowett, A., Kagan, C., Karanika-Murray, M., Kaye, L.K., Kumari, V., Lewandowsky, S., Lightman, S., Malpass, D., Meins, E., Morgan, B.P., Morrison Coulthard, L.J., Reicher, S.D., Schacter, D.L., Sherman, S.M., Simms, V., Williams, A., Wykes, T. and Armitage, C.J. (2020), Research priorities for the COVID-19 pandemic and beyond: A call to action for psychological science. Br. J. Psychol., 111: 603-629 e12468. https://doi.org /10.1111/bjop.12468.

British Psychological Society, 2020. Delivering effective public health campaigns during Covid-19. Leicester, UK. Available from. https://www.bps.org.uk/sites/www.bps. org.uk/files/Policy/Policy\%20-\%20Files/Delivering\%20effective\%20public\%20he alth\%20campaigns\%20during\%20Covid-19.pdf.

Statistics UOfN, 2020. Available from. https://www.ons.gov.uk/peoplepopulationand community/birthsdeathsandmarriages/deaths/bulletins/deathsinvolvingcovid19byl ocalareasanddeprivation/deathsoccurringbetween1marchand31july2020.

Taylor, M.R., Agho, K.E., Stevens, G.J., Raphael, B., 2008. Factors influencing psychological distress during a disease epidemic: data from Australia's first outbreak of equine influenza. BMC Public Health 8, 347.

Thomas, E., Serwicka, I., Swinney, P., 2015. Urban demographics. In: Where People Live and Work, Centre for Cities.

Williams, S.N., Armitage, C.J., Tampe, T., Dienes, K., 2020. Public perceptions and experiences of social distancing and social isolation during the COVID-19 pandemic: a UK-based focus group study. BMJ Open 10 (7) e039334. 